\title{
A classical particle in heat bath under the influence of external noise
}

\author{
J. Mencia Bravo \\ Departamento de Matemática Aplicada III, Universidad Politécnica de Cataluña, Avd. Alcalde Rovira \\ Roure, 177, 250086 Lerida, Spain \\ R. M. Velasco \\ Departamento de Fisica, UAM Iztapalapa, 09340 México D.F., Mexico \\ J. M. Sancho \\ Departamento de Estructura y Constituyentes de la Materia, Universidad de Barcelona, Diagonal 647, \\ 08028 Barcelona, Spain
}

(Received 4 January 1989; accepted for publication 12 April 1989)

A very simple model of a classical particle in a heat bath under the influence of external noise is studied. By means of a suitable hypothesis, the heat bath is reduced to an internal colored noise (Ornstein-Uhlenbeck noise). In a second step, an external noise is coupled to the bath. The steady state probability distributions are obtained.

\section{INTRODUCTION}

Our interest and the main purpose of this paper is the study of a system under the influence of internal and external fluctuations from the microscopic point of view of Hamiltonian dynamics.

The study of a system interacting with its surroundings is an interesting problem and has deserved great attention.' The methods to derive the equations of motion of such a system have been very different; they go from the projection operator technique ${ }^{2}$ to the direct elimination of the heat bath surrounding the system. ${ }^{3-9}$ The main results arising in such methods are expressed through generalized Langevin equations or the corresponding kinetic equations for the probability density. Those methods start with a selection of the relevant variables for the system, and they eliminate the irrelevant ones to obtain the behavior of the quantities we are really interested in. The relevant variable equations of motion contain some characteristics of the irrelevant variables which manifest themselves as a noise. This is usually called internal noise.

On the other hand, the behavior of a system driven by an external noise has also been studied through the introduction of stochastic terms in the phenomenological equation of motion of the relevant variables. To be specific, let us think of the equation of motion for a relevant variable $x$,

$$
\dot{x}=v(a, x),
$$

where $a$ is the parameter which will be stochastically driven. Then Eq. (1.1) becomes

$$
\dot{x}=v(a+\mu(t), x)
$$

where $\mu(t)$ is a stochastic process or noise with well-defined statistics. Equation (1.2) is called a stochastic differential equation. The problems associated with this kind of equation have been extensively studied in the literature. ${ }^{10}$

The joint study of internal and external noises was considered by means of a master equation. ${ }^{11}$ The internal noise was scaled with the size of the system, and the external noise was introduced through the parameters of the probability transitions. This approach does not incorporate the opportunity to study the effect of thermal noise.
In this work, we are interested in the following explicit problem: A classical particle is immersed in a heat bath of normal modes under the influence of an external stochastic field. In Sec. II we study this simple system without external noise in order to obtain a new representation for the internal noise. Section III is devoted to the study of this system when the heat bath is coupled with an external delta-correlated noise, and in Sec. IV we consider the coupling with an Ornstein-Uhlenbeck (OU) external noise. In all those cases, we found the stationary solution for the probability density. Finally, we make some comments about the results we have obtained.

\section{INTERNAL NOISE}

We start with the classical problem of a particle of mass $M$ coupled to a heat bath of $N$ normal modes. The Hamiltonian is given by

$$
\begin{aligned}
H= & \frac{p^{2}}{2 M}+V(x)+\frac{1}{2} \sum_{T}^{N}\left\{\frac{p_{\alpha}{ }^{2}}{m_{\alpha}}\right. \\
& \left.+m_{\alpha} w_{\alpha}{ }^{2}\left(q_{\alpha}-a_{\alpha}(x)\right)^{2}\right\},
\end{aligned}
$$

where $x, p$ are the coordinate and the momentum of the particle, respectively, $\left(q_{\alpha}, p_{\alpha}\right)$ are the variables associated to the $\alpha$ normal mode, $w_{\alpha}$ is the corresponding frequency, and the quantity $a_{\alpha}(x)$ measures the interaction between the particle and the bath. $V(x)$ is the potential energy of the particle.

The method we will follow here was developed by Zwanzig, ${ }^{8}$ and applied to several systems by Lindenberg et $a l .{ }^{9}$ The Hamilton equations for the normal mode variables $\left(q_{\alpha}, p_{\alpha}\right)$ are immediately solved and their direct substitution in the Hamilton equations for the particle gives us ${ }^{\mathbf{8 , 9}}$

$\dot{x}=p / M$,

$\dot{p}=-V^{\prime}(x)-\frac{1}{M} \int_{0}^{t} d t^{\prime} \beta\left(t^{\prime}\right) P\left(t-t^{\prime}\right)+f_{0}(t)$,

where we have assumed that the interaction between the particle and the heat bath is linear, 


$$
a_{\alpha}(x)=\theta_{\alpha} x .
$$

The last term on the rhs of Eq. (2.3) is the fluctuating force,

$$
\begin{aligned}
f_{0}(t)= & \sum_{l}^{N} \theta_{\alpha}\left\{\left[q_{\alpha}(0)-a_{\alpha}(x(0))\right] m_{\alpha} w_{\alpha}^{2} \cos \left(w_{\alpha} t\right)\right. \\
& \left.+p_{\alpha}(0) w_{\alpha} \sin \left(w_{\alpha} t\right)\right\} .
\end{aligned}
$$

It depends on the coupling function $\theta_{\alpha}$ and the normal mode initial conditions, which we will assume to be canonically distributed. The statistical properties of this fluctuating force are determined according to that assumption. At first we notice that $f_{0}(t)$ has a Gaussian distribution function with zero mean, and the correlation satisfies a fluctuationdissipation relation,

$$
\begin{aligned}
& \left\langle f_{0}(t) f_{0}\left(t^{\prime}\right)\right\rangle=K_{\mathrm{B}} T \beta\left(t-t^{\prime}\right), \\
& \beta(t)=\sum_{l}^{N} m_{\alpha} w_{\alpha}{ }^{2} \theta_{\alpha}{ }^{2} \cos \left(w_{\alpha} t\right) .
\end{aligned}
$$

It is well known that a nonlinear interaction between the normal modes and the particle leads us to an equation with multiplicative noise. ${ }^{9}$ But here we are much more interested in the analysis of internal noise properties. In order to accomplish this fact, we realize that the number of the normal modes of the heat bath should be very large $(N \gg 1)$, and we will also assume that the frequencies are distributed according to a Lorentzian function. This assumption resembles broadly the behavior of the hydrodynamical modes in a macroscopic system. ${ }^{12}$

The frequency distribution we propose is

$$
g(w)=2 N / \pi \tau\left(w^{2}+\tau^{-2}\right),
$$

where $\tau^{-1}$ is the cutoff frequency and the distribution is normalized to the number of normal modes.

The coupling function $\theta_{\alpha}=\theta(w)$ is assumed to scale with the number of oscillators $N$ in such a way that the final results were independent of $N$,

$$
\theta(w)=\theta_{0} / \sqrt{N \tau} w\left(\theta_{0} \text { is a constant }\right) .
$$

The masses $m_{\alpha}$ are put all equal to $m$. The preceding assumptions transform our internal noise $f_{0}(t)$ in an OU process. In particular, the correlation function (2.6) is obtained transforming the sum in (2.7) in a $w$ integral using (2.8) as a weight. The explicit expression we get is

$$
\left\langle f_{0}(t) f_{0}\left(t^{\prime}\right)\right\rangle=\left(m K_{\mathrm{B}} T \theta_{0}^{2} / \tau\right) e^{-\left|t-t^{\prime}\right| / \tau},
$$

where $m K_{\mathrm{B}} T \theta_{0}^{2}$ is the intensity of this noise and $\tau$ (the inverse of the cutoff frequency) is its correlation time.

It is well known that the OU noise becomes a deltacorrelated noise when the correlation time $\tau$ goes to zero, i.e., when the relaxation time of the normal modes is very small compared with the macroscopic time of the particle.

The set of Langevin equations (2.2), (2.3) and the statistical properties of the internal noise (2.10) define a nonMarkovian problem. To circumvent this difficulty, we use the well-known procedure of expanding the variables' space in order to have a set of equations without the memory function and a delta-correlated noise. ${ }^{13,14}$

An equivalent set of equations to (2.2), (2.3), and (2.10) can be written as

$$
\begin{aligned}
& \dot{x}=p / M, \\
& \dot{p}=-V^{\prime}(x)+R(t), \\
& \dot{R}=-\frac{R}{\tau}-\frac{m \theta_{0}{ }^{2}}{M \tau} p+\frac{\Gamma(t)}{\tau},
\end{aligned}
$$

where

$$
R(t)=-\frac{1}{M} \int_{0}^{t} d t^{\prime} \beta\left(t-t^{\prime}\right) P\left(t^{\prime}\right)+f_{0}(t)
$$

$R(t)$ is an additional variable and $\Gamma(t)$ is a Gaussian, zero mean, and delta-correlated noise,

$$
\left\langle\Gamma(t) \Gamma\left(t^{\prime}\right)\right\rangle=2 m K_{\mathrm{B}} T \theta_{0}^{2} \delta\left(t-t^{\prime}\right) .
$$

The evolution equation for the probability density $P(x, p, R ; t)$ is the following Fokker-Planck equation:

$$
\begin{aligned}
\frac{\partial P}{\partial t}= & -\frac{p}{M} \frac{\partial P}{\partial x}+\left(V^{\prime}(x)-R\right) \frac{\partial P}{\partial p} \\
& +\frac{\partial}{\partial R}\left(\frac{R}{\tau}+\frac{m \theta_{0}^{2}}{M \tau} p\right) P \\
& +K_{\mathrm{B}} T \frac{m \theta_{0}^{2}}{\tau^{2}} \frac{\partial^{2}}{\partial R^{2}} P .
\end{aligned}
$$

The stationary solution $P_{\mathrm{st}}(x, P, R)$ is given by

$$
P_{\mathrm{st}}(x, p, R) \approx \exp \left\{-\frac{1}{K_{\mathrm{B}} T}\left(\frac{p^{2}}{2 M}+V(x)+\frac{\tau R^{2}}{2 m \theta_{0}^{2}}\right)\right\} .
$$

It is a canonical distribution corresponding to the heat bath temperature, modified by the characteristic of the internal noise and the coupling of the heat bath with the particle through the variable $R(t)$. In spite of those couplings, it is also easy to prove that they are not relevant to studying the statics of the particle, because the variable $R(t)$ can be eliminated by a simple integration

$$
\begin{aligned}
P_{\mathrm{st}}(x, p) & =\int d R P_{\mathrm{st}}(x, p, R) \\
& \approx \exp \left\{-\frac{1}{K_{\mathrm{B}} T}\left(\frac{p^{2}}{2 M}+V(x)\right)\right\} .
\end{aligned}
$$

The result is the usual Maxwell-Boltzmann distribution function as we could expect. From the point of view of the stationary solution, it does not matter whether $\beta(t)$ is deltacorrelated or not. The difference between those two cases will be in the dynamics of the system.

\section{INTERNAL VERSUS EXTERNAL DELTA- CORRELATED NOISE}

We are interested now in the effects caused by the presence of an external noise. If we start considering a direct coupling between the external noise and the particle coordinates through a parameter in the potential, then we will get the same results obtained when we introduce an external noise in a system described by a generalized Langevin equation. We do not study that problem here, because its approach is standard. ${ }^{10}$

The interesting case occurs when the coupling with the external noise is through the heat bath. The interaction we will study here is also the simplest one. It is linear in the bath 
coordinates. The Hamiltonian (2.1) is modified by adding a new term,

$$
H_{\mathrm{int}}=\frac{1}{2} \sum_{I}^{N} \phi_{\alpha} q_{\alpha} \epsilon(t) .
$$

The function $\phi_{\alpha}(t)$ measures the interaction intensity and $\epsilon(t)$ is the external noise which we will assume to be Gaussian. The correlation function of $\epsilon(t)$ will be specified later.

The elimination of the heat bath variables follows the same way as in the previous section, leading to the generalized Langevin equation

$$
\begin{aligned}
\dot{x}= & p / M, \\
\dot{p}= & -V^{\prime}(x)-\frac{1}{M} \int_{0}^{t} d t^{\prime} \beta\left(t^{\prime}\right) p\left(t-t^{\prime}\right) \\
& +f_{0}(t)+\pi(t) .
\end{aligned}
$$

The quantities $\beta(t)$ and $f_{0}(t)$ are the same as in (2.5) and (2.7). Note that $\pi(t)$ is a fluctuating force related with the external noise through

$$
\pi(t)=-\int_{0}^{t} \Phi\left(t-t^{\prime}\right) \epsilon\left(t_{1}\right) d t^{\prime}
$$

where

$$
\Phi(t)=\sum_{l}^{N} \theta_{\alpha} \phi_{\alpha} w_{\alpha} \sin \left(w_{\alpha} t\right)
$$

The statistical properties of $\pi(t)$ are determined by the normal mode distribution (2.8), the couplings of the system with the bath (2.4) and the bath with the external noise (3.1), and the external noise itself.

Now the coupling function $\phi_{\alpha}=\phi(w)$ in (3.1) is also chosen to scale with the system size in order to obtain a finite result in the continuous limit,

$$
\phi(w)=\sqrt{\tau N^{-1}} w \Phi_{0} \quad\left(\Phi_{0}=\text { constant }\right) .
$$

A direct substitution of Eqs. (2.8), (2.9), and (3.6) in Eq. (3.5) leads to

$$
\Phi(t)=\left(\theta_{0} \Phi_{0} / \tau\right) e^{-t / \tau},
$$

which allows the calculation of the correlation function for the $\pi(t)$ noise. So far, the calculation we have done in this section is independent of the correlation function for the external noise $\epsilon(t)$. To continue, it is necessary to specify that function. First we will assume that $\epsilon(t)$ is a delta-correlated noise,

$$
\left\langle\epsilon(t) \epsilon\left(t^{\prime}\right)\right\rangle=2 D \delta\left(t-t^{\prime}\right) .
$$

By definition, it is independent of the internal noise properties. Then

$$
\left\langle\epsilon(t) f_{0}\left(t^{\prime}\right)\right\rangle=0 .
$$

The correlation function of $\pi(t)$ noise is immediately calculated and it is given by

$$
\left\langle\pi(t) \pi\left(t^{\prime}\right)\right\rangle=D\left(\theta_{0} \Phi_{0}\right)^{2} \tau^{-1} e^{-\left|t-t^{\prime}\right| / \tau},
$$

where we have neglected the transient terms $\left(t, t^{\prime}>\tau\right)$. Equation (3.10) shows how the heat bath dresses the external noise. Although the external noise is a delta-correlated one, the particle sees it as an external OU noise with the same correlation time as the internal noise but with an intensity depending on the couplings and the external noise intensity.

To construct the Fokker-Planck equation, we will follow the same procedure as in Sec. II with few changes. Equation (3.3) has two noises and according to Eqs. (2.10) and (3.10), both have the same correlation time. We define an effective Gaussian noise $\sigma(t)=f_{0}(t)+\pi(t)$, which has zero mean and a correlation function given by

$$
\left\langle\sigma(t) \sigma\left(t^{\prime}\right)\right\rangle=\left(m K_{\mathrm{B}} T+D \Phi_{0}{ }^{2}\right) \theta_{0}{ }^{2} \tau^{-1} e^{-\left|t-t^{\prime}\right| / \tau} .
$$

Note that $\sigma(t)$ is also an OU noise, but there is not a fluctuation dissipation relation because the external noise is present.

The set of equivalent Markovian equations is the same as (2.11) but the intensity of the delta-correlated noise is given now by $\left(m K_{\mathrm{B}} T+D \Phi_{0}{ }^{2}\right) \theta_{0}{ }^{2}$, which changes the diffusion coefficient in the corresponding Fokker-Planck equation,

$$
\begin{aligned}
\frac{\partial P}{\partial t}= & -\frac{p}{M} \frac{\partial P}{\partial x}+\left(V^{\prime}(x)-R\right) \frac{\partial p}{\partial \rho} \\
& +\frac{\partial}{\partial R}\left(\frac{R}{\tau}+\frac{m \theta_{0}^{2}}{M \tau} p\right) P \\
& +\left(m K_{\mathrm{B}} T+D \Phi_{0}{ }^{2}\right) \frac{\theta_{0}{ }^{2}}{\tau^{2}} \frac{\partial^{2} P}{\partial R^{2}} .
\end{aligned}
$$

The stationary solution has the same qualitative features as Eq. (2.14), but the "temperature" has changed according to the new diffusion coefficient

$$
P_{\mathrm{st}}(x, p, R) \approx \exp \left\{-\frac{1}{K_{\mathrm{B}} T+(D / m) \Phi_{0}{ }^{2}}\left(\frac{p^{2}}{2 M}+V(x)+\frac{\tau}{2 m \theta_{0}{ }^{2}} R^{2}\right)\right\}
$$

This is a canonical distribution with an effective diffusion which depends on the external noise intensity and the coupling between the noise and the heat bath.

Once again the correlation time $\tau$ coming from the internal noise is irrelevant to the statics because the integration in the additional $R$ variable is not coupled to the variables $(x, p)$, giving us the usual exponential distribution function (2.15) but with a new diffusion coefficient.

\section{INTERNAL VERSUS EXTERNAL ORNSTEIN- UHLENBECK NOISE}

In this section, we consider that the external noise is an OU process with a correlation function given by

$$
\left\langle\epsilon(t) \epsilon\left(t^{\prime}\right)\right\rangle=\left(D / \tau^{\prime}\right) e^{-\left|t-t^{\prime}\right| / \tau^{\prime}},
$$

where $D$ and $\tau^{\prime}$ are the intensity and the correlation time, 
respectively. A direct calculation gives us the correlation function of $\pi(t)$,

$$
\begin{aligned}
\left\langle\pi(t) \pi\left(t^{\prime}\right)\right\rangle= & \frac{D\left(\theta_{0} \Phi_{0}\right)^{2}}{\left(\tau^{\prime} / \tau\right)^{2}-1} \frac{\tau^{\prime}}{\tau}\left\{\frac{1}{\tau} \exp \left(-\frac{\left|t-t^{\prime}\right|}{\tau^{\prime}}\right)\right. \\
& \left.-\frac{1}{\tau^{\prime}} \exp \left(-\frac{\left|t-t^{\prime}\right|}{\tau}\right)\right\},
\end{aligned}
$$

where we have neglected the transient terms. The dressed external noise $\pi(t)$ now has a more complicated correlation function with two correlation times, $\tau$ and $\tau^{\prime}$. In order to see what is the meaning of Eq. (4.2), let us assume that the external correlation time is bigger than the internal one. Then the dressed noise is dominated by the external noise,

$$
\left\langle\pi(t) \pi\left(t^{\prime}\right)\right\rangle=\left[D\left(\theta_{0} \Phi_{0}\right)^{2} / \tau^{\prime}\right] e^{-\left|z-z^{\prime}\right| / \tau^{\prime}} .
$$

On the other hand, when the external correlation time is smaller than the internal one, we then recover (3.10).

Now let us see what is the physical situation corresponding to (4.3). The heat bath degrees of freedom act on the system as an internal noise, and relax with a characteristic time $\tau$. A physical intuition leads us to think that the internal degrees of freedom should relax faster than any real external noise. So we will take a delta-correlated internal noise $(\tau=0)$, and then the dressed noise correlation is dominated by the external noise (4.3). The set of Langevin equations (3.2) and (3.3) is simplified,

$$
\begin{aligned}
& \dot{x}=p / M, \\
& \dot{p}=-V^{\prime}(x)-\left(m \theta_{0}^{2} / M\right) p+f_{0}(t)+\pi(t),
\end{aligned}
$$

where we have taken the white noise limit for $f_{0}(t)[\tau=0$ in (2.10)].

$$
\begin{aligned}
& \text { Note that } \\
& \left\langle f_{0}(t) f_{0}\left(t^{\prime}\right)\right\rangle=2 K_{\mathrm{B}} \operatorname{Tm} \theta_{0}{ }^{2} \delta\left(t-t^{\prime}\right)
\end{aligned}
$$

and the term $-m \theta_{0}^{2} p / M$ in Eq. (4.4) comes from the memory function which is now $\beta\left(t-t^{\prime}\right)=2 m \theta_{0}^{2} \delta\left(t-t^{\prime}\right)$.

To study the problem described by the set of Eqs. (4.4), we define an effective Gaussian OU noise,

$$
\Omega(t)=f_{0}(t)+\pi(t),
$$

with an intensity $D_{R}$ and a correlation time $\tau_{R}$ given by

$$
\begin{aligned}
& D_{R}=\int_{0}^{\infty}<\Omega(t) \Omega(0) d t, \\
& \tau_{R}=\frac{1}{D_{R}} \int_{0}^{\infty} t<\Omega(t) \Omega(0) d t .
\end{aligned}
$$

These definitions give

$$
\begin{aligned}
& D_{R}=m \theta_{0}{ }^{2}\left(K_{\mathrm{B}} T+D \Phi_{0}{ }^{2} / m\right), \\
& \tau_{R}=D\left(\theta_{0} \Phi_{0}\right)^{2} \tau^{\prime} / D_{R},
\end{aligned}
$$

which are some combinations of the parameters characterizing the noises and their interactions. The problem has been reduced to a set of equations with an effective $O U$ noise. The stationary solution has been discussed in the literature. ${ }^{15}$

The stationary distribution is now

$$
P_{\mathrm{st}}(x, p) \approx \exp \left\{-\frac{p^{2}}{D_{p} 2 M}+\frac{V(x)}{D_{x}}\right\},
$$

where

$$
D_{p}=\frac{D_{R}}{m \theta_{0}^{2}\left(1+m \theta_{0}^{2} \tau_{0} / M\right)}, \quad D_{x}=\frac{D_{R}}{m \theta_{0}^{2}} .
$$

Now we have different values for the $p$-diffusion and the $x$ diffusion coefficients. We notice that the $D_{x}$ coefficient is the same as in Eq. (3.15), but the $D_{p}$ coefficient has changed again and it shows a correction due to the external noise correlation time. Finally, we mention that $\tau^{\prime}$ can not be eliminated as it was done in earlier cases. The interaction between internal and external noises will now effect the static properties of our system.

\section{CONCLUDING REMARKS}

Here we summarize the results obtained in this paper. At first we considered the simplest Hamiltonian system to study the behavior of an open subsystem immersed in a heat bath. The heat bath coordinates were eliminated and we found the way to model an Ornstein-Uhlenbeck noise. In this case, we have obtained that the internal noise correlation time is not a relevant quantity in the stationary solution of the Fokker-Planck equation. The delta-correlated external noise, coupled with the heat bath and with an OU internal noise, also has that property. But the diffusion coefficient was modified by the coupling parameters, and as a consequence there is not a fluctuation-dissipation relationship.

From the point of view of a more realistic situation, we have found that the most interesting case corresponds to a delta-correlated internal noise and an OU external noise. The stationary solution has two renormalized diffusion coefficients, one of them depending explicitly of the external noise correlation time. Obviously the fluctuation-dissipation relationship does not hold, because the external noise is present.

The approach presented here opens the possibility of studying more complicated systems with nonlinear couplings.

\section{ACKNOWLEDGMENTS}

J.M.B. thanks M. San Miguel for helping in his introduction to this field.

Partial financial support from the program CONACYT-CSIC (Mexico-Spain) is acknowledged. Two of us (J.M.B. and J.M.S.) also acknowledge the financial support of Direccíon General de Investigacíon Científica y Técnica, Project no. PR87-0014 (Spain).

'R. Zwanzig, in Lectures in Theoretical Physics, edited by W. E. Brittin, B. W. Downs, and J. Downs (Interscience, New York, 1961), Vol.3; H. Mori, Prog. Theor. Phys. 34, 423 (1965).

${ }^{2}$ R. Zwanzig, Phys. Rev. 124, 983 (1961).

${ }^{3} \mathrm{H}$. Grabert, Projection Operator Techniques in Nonequilibrium Statistical Mechanics (Springer, Berlin, 1982).

${ }^{4} \mathrm{P}$. Grigolini, in Advances in Chemical Physics, edited by M. W. Evans, P. Grigolini, and G. Pastori Parravicini (Wiley, New York, 1985).

${ }^{5}$ N. G. Van Kampen and I. Oppenheim, Physica A 138, 231 (1986).

6J. J. Brey, J. M. Casado, and M. Morillo, Physica A 121, 122 (1984).

${ }^{7}$ G. W. Ford, M. Kac, and P. Mazur, J. Math. Phys. 6, 504 (1965).

${ }^{8}$ R. Zwanzig, J. Stat. Phys. 9, 215 (1973).

${ }^{9} \mathrm{~K}$. Lindenberg and V. Seshadri, Physica A 109, 483 (1981); V. Seshadri and $\mathrm{K}$. Lindenberg, ibid. A 115, 501 (1982). 
${ }^{10}$ W. Horsthemke and R. Lefever, Noise Induced Transitions (Springer, Berlin, 1984).

"M. San Miguel and J. M. Sancho, Phys. Lett. A 90, 455 (1982); J. M. Sancho and M. San Miguel, J. Stat. Phys. 37, 151 (1984).

${ }^{12} \mathrm{P}$. Resibois and M. de Leener, Classical Kinetic Theory of Fluids (Wiley-
Interscience, New York, 1977).

${ }^{13}$ F. Marchesoni and P. Grigolini, J. Chem. Phys. 78, 6287 (1983).

${ }^{14} \mathrm{H}$. Risken, The Fokker-Planck Equation, Methods of Solution and Applications (Springer, Berlin, 1984)

${ }^{15}$ L. Ramirez-Piscina and J. M. Sancho, Phys. Rev. A 37, 4469 (1988). 